\title{
QUEEN'S
UNIVERSITY
BELFAST
}

\section{Online measurements of gamma radiation from laser-plasma and signal unfolding using a scintillator calorimeter}

Istokskaia, V., Stránský, V., Giuffrida, L., Versaci, R., Olšovcová, V., Singh, S., Krupka, M., Dudžák, R., Krása, J., \& Margarone, D. (2021). Online measurements of gamma radiation from laser-plasma and signal unfolding using a scintillator calorimeter. In S. S. Bulanov, J. Schreiber, \& C. B. Schroeder (Eds.), Laser Acceleration of Electrons, Protons, and lons VI [117790D] (Proceedings of SPIE - The International Society for Optical Engineering; Vol. 11779). SPIE - The International Society for Optical Engineering. https://doi.org/10.1117/12.2590815

Published in:

Laser Acceleration of Electrons, Protons, and Ions VI

\section{Document Version:}

Peer reviewed version

Queen's University Belfast - Research Portal:

Link to publication record in Queen's University Belfast Research Portal

\section{Publisher rights}

Copyright 2021 SPIE. This work is made available online in accordance with the publisher's policies. Please refer to any applicable terms of use of the publisher.

\section{General rights}

Copyright for the publications made accessible via the Queen's University Belfast Research Portal is retained by the author(s) and / or other copyright owners and it is a condition of accessing these publications that users recognise and abide by the legal requirements associated with these rights.

Take down policy

The Research Portal is Queen's institutional repository that provides access to Queen's research output. Every effort has been made to ensure that content in the Research Portal does not infringe any person's rights, or applicable UK laws. If you discover content in the Research Portal that you believe breaches copyright or violates any law, please contact openaccess@qub.ac.uk. 


\title{
Online measurements of gamma radiation from laser-plasma and signal unfolding using a scintillator calorimeter
}

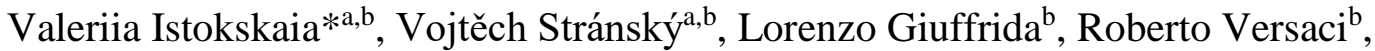 \\ Veronika Olšovcováb, S. Singh ${ }^{\mathrm{c}, \mathrm{d}}$, M. Krupka ${ }^{\mathrm{a}, \mathrm{c}, \mathrm{d}}$, R. Dudžák ${ }^{\mathrm{d}}, \mathrm{J}_{\text {. Krása }}^{\mathrm{d}}$, and Daniele Margarone ${ }^{\mathrm{b}, \mathrm{e}}$ \\ ${ }^{\mathrm{a} C z e c h}$ Technical University in Prague, Faculty of Nuclear Sciences and Physical Engineering, \\ Prague, Czech Republic; 'bLI Beamlines, Institute of Physics of the Czech Academy of Sciences, \\ Dolní Břežany, Czech Republic; ' Institute of Plasma Physics of the Czech Academy of Sciences, \\ Prague, Czech Republic; ' Institute of Physics of the Czech Academy of Sciences, \\ Prague, Czech Republic; ' $C$ Centre for Plasma Physics, School of Mathematics and Physics, Queen's \\ University Belfast, Belfast, United Kingdom
}

\begin{abstract}
With the development of high-intensity and high-repetition-rate laser systems, it has become crucial to be able to measure and characterize the high-energy gamma radiation from laser-matter interaction in real-time. Therefore, a scintillator-based electromagnetic calorimeter aimed at high-energy electron and photon detection under high-repetition rate is being developed at the ELI Beamlines facility. Together with an ad hoc created unfolding technique, it is possible to reconstruct energies/temperatures of one or two thermal populations present in the radiation. A preliminary test of the device performed at the PALS experimental facility together with the corresponding signal unfolding is here presented.
\end{abstract}

Keywords: calorimeter, scintillators, data processing methods, gamma radiation, laser-plasma characterization

\section{INTRODUCTION}

The interaction between a powerful laser pulse and a target induces the material ionization and the resultant plasma creation that leads to the emission of a copious number of particles and radiation ${ }^{1}$. The laser energy is typically transferred via collisionless processes ${ }^{2}$ to the so-called hot electrons (superthermal population of electrons ${ }^{3,4}$ ) that penetrate through the plasma and decelerate producing Bremsstrahlung radiation. Detection of such radiation can provide with important insight to laser-plasma interaction, especially to absorption processes, hot electrons temperature and their transport in plasma ${ }^{2,5,6}$.

However, in the case of PW lasers, the Bremsstrahlung radiation is highly energetic (hundreds of $\mathrm{keV}^{-} \mathrm{several} \mathrm{MeV}^{7,8}$ ), falling within the gamma range, and it is emitted in short flashes ( $\mathrm{fs}-\mathrm{ps}$ range $^{9}$ ). Moreover, the radiation yield is extremely large per each flash, making single-photon counting based diagnostics not suitable for such measurements. Among the most advantageous detectors for energetic and pulsed radiation are various spectrometers utilizing a FUJIfilm image plate ${ }^{10,11}$ as a radiation-sensitive part ${ }^{12-14}$. However, such passive devices are not applicable to the highrepetition-rate working regime since the image plate must be replaced after each shot and requires a sophisticated and time-consuming post-processing.

Detectors based on scintillators arranged in a stack and coupled with optical readout seem to offer a novel approach to measuring such Bremsstrahlung radiation, as was demonstrated in the literature ${ }^{15-17}$. Scintillating materials emit visible light, detectable by a standard CMOS or CCD camera, when exposed to the ionizing radiation. The amount of light is proportional to the radiation flux. Short scintillation duration enables to perform measurements online during highrepetition-rate experiment.

In this paper, we present an online scintillator-based electromagnetic calorimeter (EMC) developed at the ELI Beamlines laser facility ${ }^{18}$. The device is designed to work in a repetition rate up to $50 \mathrm{~Hz}$ and measure high-energy radiation (up to 
tens of $\mathrm{MeV}$ ) generated by a high-intensity (up to $10^{22} \mathrm{~W} / \mathrm{cm}^{2}$ ) laser system, such as the 1 PW HAPLS laser ${ }^{19}$ available at ELI Beamlines. The following sections contain the description of the detector design and the signal unfolding technique, along with demonstration of some of the preliminary experimental tests.

\section{DETECTOR DESIGN AND SIGNAL UNFOLDING}

The electromagnetic calorimeter is a stack of 15 crystals of different thickness made of two scintillator types. The usage of two scintillating materials arises from the assumption of double electron population in the laser-plasma - thermal and superthermal. Both populations are expected to have distant characteristic temperatures (low-temperature and hightemperature components) and follow the Maxwell-Boltzmann (MB) energy distribution temperatures ${ }^{20,21}$. Usage of scintillators of different density is supposed to separate the Bremsstrahlung radiation coming from both electrons components and measure it simultaneously by one device.

A sketch of the detector is shown in Figure 1, a). The frontal part of it is composed of the EJ200 plastic scintillators (1.03 $\mathrm{g} / \mathrm{cm}^{3}, 10^{4}$ photons/MeV). Given their low density, plastic crystals are supposed to stop the low-energy components of the radiation. The second part of the device is comprised of the Bismuth Germinate (BGO) scintillators $\left(7.13 \mathrm{~g} / \mathrm{cm}^{3}\right.$, $8 \times 10^{3}$ photons $/ \mathrm{MeV}$ ) and is aimed at measuring the high-energy radiation component due to the high density. The scintillators are inserted into a specially developed black plastic holder, ensuring a proper light separation between the adjacent crystals.

The dimensions of the EJ200 scintillators are 20x10x20 $\mathrm{mm}^{3}$. The middle BGO crystals, located after the plastic part, are thinner $\left(20 \times 5 \times 20 \mathrm{~mm}^{3}\right)$ in order to increase the energy resolution in the most exposed region. The very last four BGOs are $20 \times 10 \times 20 \mathrm{~mm}^{3}$, similarly to the EJ200. The signal readout is implemented by a CMOS Manta 235B camera coupled with a suitable small focal length lens. The whole setup has to be covered by an optical shielding in order to prevent the external light to interfere with the scintillation. In our setup, it was implemented by using double layers of a dense black fabric.
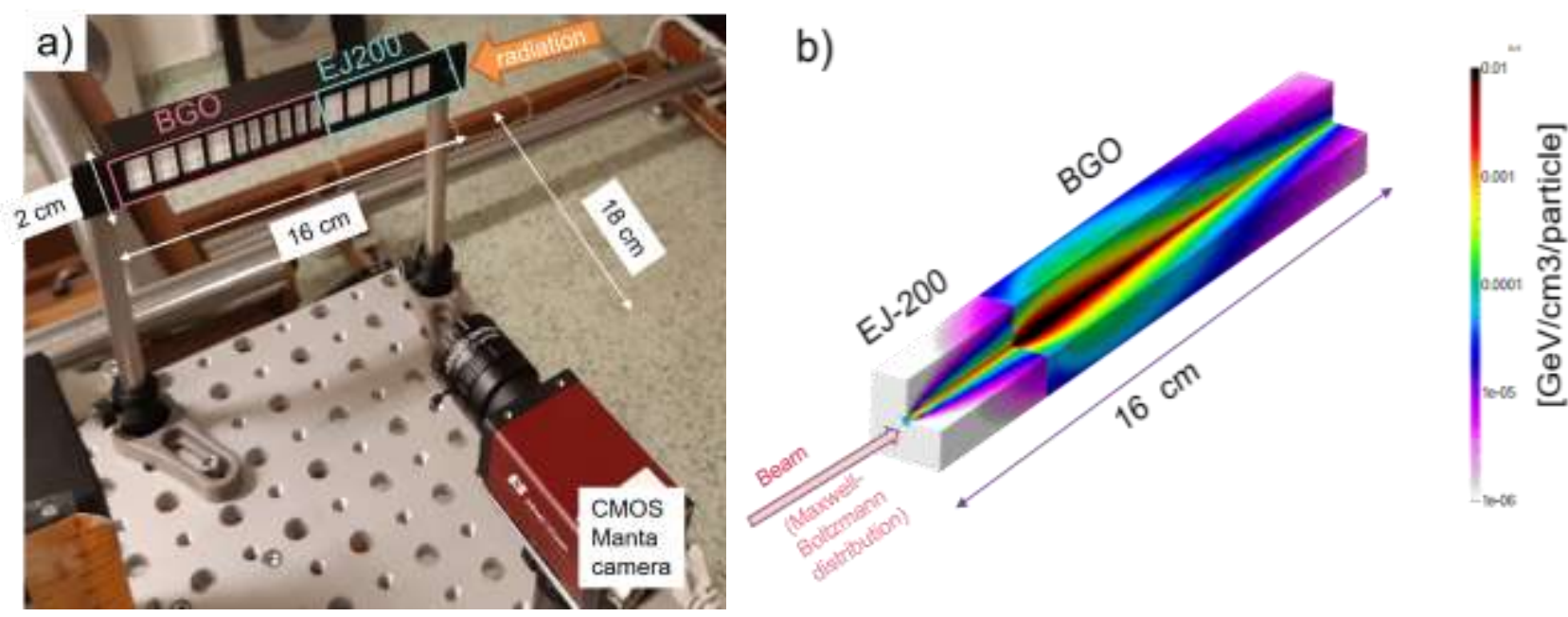

Figure 1. a) Electromagnetic calorimeter setup: scintillating crystals of two kinds (EJ200 and BGO) are placed in a plastic holder the optical signal from the crystals is recorded by a CMOS Manta camera. The radiation is incident from the EJ200 crystals side. The setup dimensions are also sketched here. Optical shielding is not shown in the figure. b) An illustrative example of FLUKA simulations of the radiation energy deposition along the calorimeter. A simplified detector model is built in the Flair software.

A specially developed signal unfolding technique enables to reconstruct one or two temperatures of the radiation. The interaction between the radiation and the detector is simulated using FLUKA ${ }^{22-24}$ to score the deposited energy along the device, in each of the crystals. Figure 1, b) demonstrates an illustrative example of the simulated energy deposition using a simplified detector design built using Flair software ${ }^{25}$. As a result, a response matrix is created for a range of predefined MB temperatures of the incoming radiation. The response matrix is a key component for the unfolding technique: the measured signal is compared with the simulated one aiming at minimization of their difference $\left(\chi^{2}-\right.$ 
function) by undergoing a series of steps. Usage of a nonlinear regression model allows to obtain the standard error of the unfolded temperature and statistical parameters such as p-value and adjusted R-squared ${ }^{26}$, in order to evaluate whether the unfolding output is trustable. Full description of the unfolding technique can be found in reference 27 . The uncertainty of determining a MB temperature was estimated in reference 28 to be approximately $15 \%$. For an ordinary commercial off-the-shelf computer, the execution time of the unfolding algorithm is approximately $10 \mathrm{~s}$ or $100 \mathrm{~s}$ for one or two temperature searches, respectively.

\section{EXPERIMENTAL TESTS AT PALS}

One of the preliminary experimental tests of the electromagnetic calorimeter aimed at laser-plasma generated Bremsstrahlung measurements took place at Prague Asterix Laser System (PALS) facility ${ }^{29}$, using an iodine laser operated in a single-pulse regime at $1315 \mathrm{~nm}$, delivering a maximum laser energy of $620 \mathrm{~J}$ in about $350 \mathrm{ps.} \mathrm{During} \mathrm{the}$ experiment, the laser was focused down to moderate laser intensities of $\sim 3 \times 10^{16} \mathrm{~W} / \mathrm{cm}^{2}$ ) and characterized with the focal spot size of approximately $100 \mu \mathrm{m}$. Various metal targets were used in the experiment.

The experimental setup is sketched in Figure 2. Two identical calorimeters, constructed according to the description in Figure $1 \mathrm{a}$ ), were placed at two different positions in front of glass viewports outside of the vacuum chamber. The viewports were located at $0^{\circ}$ and $30^{\circ}$ to the target normal and the calorimeters were aligned accordingly. Given the outside-of-the-vacuum location, a possible impact of such particles as electrons and ions on the device was eliminated in this experiment. Collimators of $5 \mathrm{~cm}$ thickness and $1.5 \times 1.5 \times 5 \mathrm{~cm}^{3}$ holes dimensions were built using lead bricks in front of each detector in order to minimize the impact of backscattered radiation on detectors' sides. The lead served also as a shielding for the readout cameras in order to reduce the direct irradiation on cameras' chips.

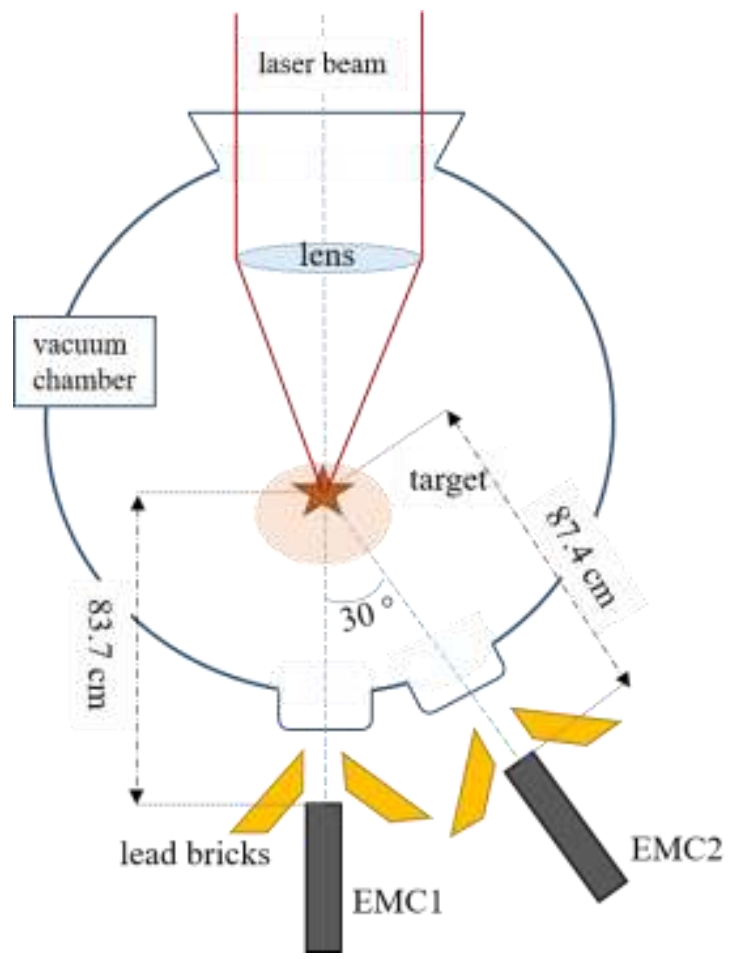

Figure 2. Sketch of the experimental setup during the PALS experiment. Two calorimeters labelled EMC1 and EMC2 are located outside of the vacuum chamber at different angels $\left(0^{\circ}\right.$ and $\left.30^{\circ}\right)$ to the target normal. Lead collimators are placed in front of the detectors to reduce the secondary radiation. 
In this paper, we further describe results for a $15-\mu \mathrm{m} \mathrm{Pb}$ target only. Two measured signals presented in Figure 3 correspond to the same shot; the laser energy reached $535 \mathrm{~J}$ in this case. On the top of the figure, the post-processed signal images for both detectors are demonstrated. As can be seen, the strongest signal on both images is shown by the first BGO crystal. The incoming photons interacted slightly and gradually with the light plastic part, depositing most of their energy once they entered the denser BGO region. On the other hand, the radiation flux seemed not to be strong enough to trigger a comparable scintillation in the remaining BGO crystals.

The measured signal and the reconstructed one obtained using the unfolding technique are compared on the bottom of Figure 3 and shown as red and blue lines, respectively. The left part of the figure corresponds to the $0^{\circ}$ location (a), while the right one corresponds to the $30^{\circ}$ location (b). The error bars are given as a standard mean error calculated for each crystal area. Due to the low signal-to-noise ratio in the last three crystals, that accounts for the low radiation flux in the area, only the first 12 crystals were taken into consideration during the unfolding. For the calorimeter located on the target normal $\left(0^{\circ}\right)$, the unfolded temperature was found as $(51.5 \pm 2.9) \mathrm{keV}$, while the corresponding statistical parameters amounted to $8.3 \mathrm{e}-09$ for $\mathrm{p}$-value and 0.999 for the adjusted R-squared, indicating that the result is in agreement with the nonlinear model. For the second detector, the unfolded temperature is $(47.6 \pm 4.7) \mathrm{keV}$, with $\mathrm{p}$-value $=1.3 \mathrm{e}-06$ and the adjusted R-squared $=0.998$. The stated unfolding errors correspond only to the mathematical uncertainty of the unfolding procedure. The real uncertainty of the calorimeter is approximately $15 \%$ of the unfolded results. For the calorimeter located at $30^{\circ}$ to the target normal, the unfolding error is higher due to the weaker signal and thus lower signal-to-noise ratio. Nevertheless, both results are in a good agreement.

It is worth noting, that despite the unfolding algorithm searched for two simultaneous radiation temperatures, only one was considered to be statistically significant. Most probably, the second thermal population was either substantially less representative or less energetic, thus falling below the detection limit of the calorimeter $(10 \mathrm{keV})$.

According to the electron spectrometer data that was used during the previous PALS experiments performed under similar experimental conditions ${ }^{30,31}$, electron temperatures usually fell within the $(80-150) \mathrm{keV}$ range. Assuming that the Bremsstrahlung temperature should be similar or less than that of the electrons, our findings are in accordance with the expectations. Our previous test of the calorimeter ${ }^{27}$ using thin Ta target under similar experimental conditions indicated the same range of temperatures $(60 \mathrm{keV})$.

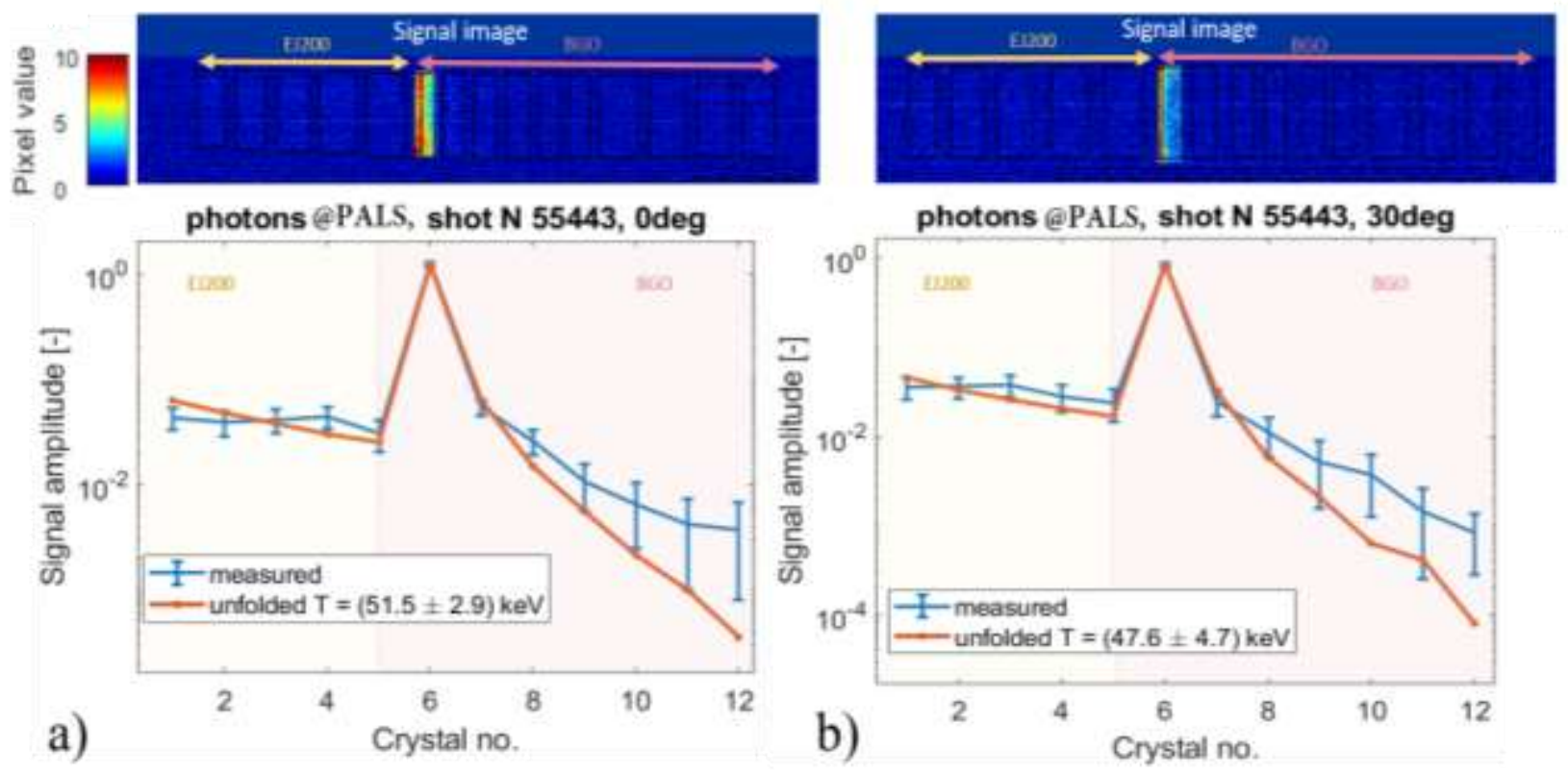

Figure 2. a)EMC1@ $0^{\circ}$ to the target normal. Top: a post-processed signal image, demonstrating the scintillation of the crystals. Bottom: comparison between the measured signal (blue line) and the result of the unfolding algorithm (red line). b) EMC2@30 to the target normal, analogously to a). 


\section{CONCLUSIONS AND PERSPECTIVE}

Two scintillator-based electromagnetic calorimeters coupled with CMOS cameras were tested in a laser-plasma experiment at the PALS facility. Using such devices, it was possible to measure Bremsstrahlung radiation generated by the hot electrons in laser-plasma. The calorimeters proved to work successfully in online regime, enabling to perform measurements on shot-to-shot basis. The ad hoc developed unfolding technique was used to reconstruct radiation temperature within a short time during the experiment.

However, during the described preliminary experimental test, the measured temperatures were about one/two orders of magnitude less than those for which the detector was optimized (several MeV - tens of MeV), resulting in a relatively weak signal. Moreover, only one temperature component was found to be significant enough to be detected, despite two components were searched for. Nevertheless, the unfolding results were consistent for both detectors and are in agreement with the expectations for the particular experimental conditions.

Future tests of the developed calorimeter at more powerful laser facilities producing high-energy Bremsstrahlung radiation are scheduled. The unfolding algorithm optimization is ongoing, aiming at the shortening of the execution time and refinement of the two-temperature search.

\section{ACKNOWLEDGEMENTS}

This work has been supported by the Ministry of Education, Youth and Sports of the Czech Republic (No. LM2018114 Prague Asterix Laser System and No. EF16_013/0001552), by the project "Advanced Research Using High Intensity Laser Produced Photons and Particles" (CZ.02.1.01/0.0/0.0/16_019/0000789), by the Access to the PALS RI under the EU LASERLAB IV project (Grant Agreement No. 654148), and by the Czech Science Foundation (Grant Nos.19. 02545S). The authors gratefully acknowledge the support of the PALS technical staff.

\section{REFERENCES}

[1] C. N. Danson et al., "Petawatt and exawatt class lasers worldwide," High Power Laser Science and Engineering, 7, 2019.

[2] T. Liseykina, P. Mulser, \& M. Murakami, "Collisionless absorption, hot electron generation, and energy scaling in intense laser-target interaction", Physics of Plasmas, 22(3):033302, 2015.

[3] S. C. Wilks, W.L. Kruer, M. Tabak and A.B. Langdon, "Absorption of ultra-intense laser pulses", Physical Review Letters, 69:1383, 1992.

[4] M. G. Haines et al., "Hot-electron temperature and laser-light absorption in fast ignition", Physical Review Letters, 102:045008, 2009.

[5] C.D. Armstrong et al., "Bremsstrahlung emission profile from intense laser-solid interactions as a function of laser focal spot size", Plasma Physics and Controlled Fusion, 61:034001, 2019.

[6] G. Hernández and F. Fernández, "Diagnostic of electron temperature from bremsstrahlung in overdense targets", Journal of Applied Physics B, 124:135, 2018.

[7] S. Palaniyappan et al., "MeV bremsstrahlung X rays from intense laser interaction with solid foils", Laser and Particle Beams, 36:502, 2018.

[8] B. Borm et al., "Properties of laser-driven hard x-ray sources over a wide range of laser intensities", Physics of Plasmas, 26:023109, 2019.

[9] H. Sawada et al., "Characterization of intense laser-produced fast electrons using hard x-rays via bremsstrahlung." Journal of Physics B: Atomic, Molecular and Optical Physics, 48.22:224008, 2015.

[10] FUJI film official website, http://www.fujifilm.com/.

[11] S. Singh, et al., "Absolute calibration of imaging plate detectors for electron kinetic energies between $150 \mathrm{kev}$ and 1.75 Mev," Review of Scientific Instruments ,88: 075105, 2017.

[12] D. Haden et al., "High energy X-ray Compton spectroscopy via iterative reconstruction", Nucl. Instrum.Meth. A 951 (2020) 163032. 
[13] S. Singh et al., "Compact high energy x-ray spectrometer based on forward Compton scattering for high intensity laser plasma experiments", Review of Scientific Instruments, 89:085118, 2018.

[14]R. Scott et al., "Measuring fast electron spectra and laser absorption in relativistic laser-solid interactions using differential bremsstrahlung photon detectors", Review of Scientific Instruments, 84:083505, 2013.

[15]D.R. Rusby et al., "Novel scintillator-based x-ray spectrometer for use on high repetition laser plasma interaction experiments", Review of Scientific Instruments, 89:073502, 2018.

[16] K. T. Behm et al., "A spectrometer for ultrashort gamma-ray pulses with photon energies greater than $10 \mathrm{MeV}$, Review of Scientific Instruments, 89.11:113303, 2018.

[17]S. Singh et al. "Bremsstrahlung emission and plasma characterization driven by moderately relativistic laserplasma interactions." Plasma Physics and Controlled Fusion, 63.3:035004, 2021.

[18] ELI Beamlines official website, https://www.eli-beams.eu/.

[19] E. Sistrunk et al., "All diode-pumped, high-repetition-rate advanced petawatt laser system (HAPLS), in CLEO: Science and Innovations", Optical Society of America, 2017.

[20] O. N. Rosmej et al., "Generation of keV hot near-solid density plasma states at high contrast laser-matter interaction”, Physics of Plasmas, 25:083103, 2018.

[21]C. Zulick et al., "High resolution bremsstrahlung and fast electron characterization in ultrafast intense lasersolid interactions", New Journal of Physics, 15, 2013.

[22] FLUKA official website, https://fluka.cern.

[23] G. Battistoni et al., "Overview of the FLUKA code", Annals of Nuclear Energy, 82:10, 2015.

[24]T.T. Böhlen et al., "The FLUKA Code: Developments and Challenges for High Energy and Medical Applications", Nucler Data Sheets, 120:211, 2014.

[25] V. Vlachoudis, "FLAIR: A Powerful But User Friendly Graphical Interface For FLUKA", in Proc. Int. Conf. on Mathematics, Computational Methods \& Reactor Physics (M\&C 2009), Saratoga Springs, New York, 2009.

[26] Y. Dodge, The Concise Encyclopedia of Statistics, Springer, 2008.

[27] V. Istokskaia et al., "Experimental tests and signal unfolding of a scintillator calorimeter for laser-plasma characterization", Journal of Instrumentation, 16(02):T02006, 2021.

[28] V. Stránský et al., "Development, optimization, and calibration of an active electromagnetic calorimeter for pulsed radiation spectrometry", submitted to Journal of Instrumentation.

[29] K. Jungwirth et al., “The Prague asterix laser system”, Physics of Plasmas, 8:2495, 2001.

[30]M. Krupka et al., "Comprehensive diagnostics of hot electron emission from plasmas produced by subnanosecond terawatt laser on thin foil metal targets", Journal of Instrumentation, 14:C12003, 2019.

[31]M. Krupka et al., "Design of modular multi-channel electron spectrometers for application in laser matter interaction experiments at Prague Asterix Laser System", Review of Scientific Instruments, 92.2:023514, 2021. 